\title{
Dynamics of a two-level atom observed via an interaction-free measurement
}

\author{
A. Luis and L. L. Sánchez-Soto \\ Departamento de Óptica, Facultad de Ciencias Físicas, Universidad Complutense, 28040 Madrid, Spain
}

(Received 6 October 1998)

\begin{abstract}
We examine the evolution of a two-level atom when its population is monitored. The detection proposed is an interaction-free measurement, so that the observation occurs without photon exchange between the atom and the apparatus. It is shown that the observed dynamics exhibits features that an isolated two-level atom cannot accommodate. Such phenomena are explained in terms of an atom-apparatus effective interaction. [S1050-2947(99)00107-9]
\end{abstract}

PACS number(s): 03.65.Bz, 42.50.Dv

\section{INTRODUCTION}

The problem of detection and measurement is of fundamental importance in quantum theory. In all cases, one obtains information after the interaction of the system of interest with an observing apparatus. Due to the strong correlations established during the detection, quantum fluctuations of the apparatus disturb the isolated evolution of the system, contrary to what happens in classical physics.

Among other consequences, this implies that the evolution of a system subjected to a continual measurement differs from the original or unobserved evolution [1-4]. For certain purposes this backaction might be regarded as an undesirable consequence. However, from another point of view, this same effect offers the possibility of tailoring the evolution of the observed system [5]. For instance, measurement is often a suitable procedure for the actual generation of special states that would be very difficult to attempt by other methods [6].

In this work we examine the dynamics of a two-level atom when its level occupation is continuously measured. The scheme we will consider here is a very simple and feasible interaction-free experiment, which is based on the resonant atom-field interaction in two coupled cavities. The remarkable concept of an interaction-free measurement is also deeply rooted in the particular features of quantum detection $[7,8]$. The main idea is that it is possible to infer by optical means the state of an object seemingly without interacting with it. The alleged absence of interaction relies on a purely corpuscular description of the electromagnetic field, where no photon exchange means no interaction [9]. However, a complete quantum description of the process shows that there is in fact an actual effective interaction during the detection, as required by the unavoidable quantum backaction on the object [10].

The evolution of the observed atom should reveal explicitly this effective interaction, especially if we take into account the strong quantum nature of the observed object. Unlike other observation mechanisms, these arrangements grant that the apparatus will induce no internal transition on the atom, providing in this way a subtle and fully quantum observation, markedly different from other detection procedures. It can be expected that the effective interaction, and the ensuing modification of the atomic evolution, will be nontrivial and worth examining.
The plan of this paper is as follows. In Sec. II we describe the interaction-free arrangement devised to infer the atomiclevel occupation. First, we look for the conditions under which the inference occurs without absorption and/or emission of light. Then, we derive the remaining evolution equation for the atom-field system. In Sec. III we examine the performance of the detection and its consequences on the atomic dynamics, comparing the results with the evolution of an unobserved two-level atom. In Sec. IV we analyze the results resorting to the quantum character of the apparatus and the atom-field effective interaction.

\section{OBSERVATION ARRANGEMENT AND JOINT ATOM- APPARATUS EVOLUTION}

The object of the observation is a two-level atom whose energy eigenstates are the ground $(|g\rangle)$ and excited $(|e\rangle)$ states. In the absence of any observation, the dynamics of the atom will be governed by some Hamiltonian $H$ stemming from its interaction with a monochromatic classical radiation field of frequency $\omega_{f}$ and from its coupling with other field modes, which includes the spontaneous decay of the excited level $|e\rangle$. The explicit form of $H$ will be considered in detail later.

The observation arrangement comprises two identical cavities coupled by a beam splitter $[8,11]$, as illustrated in Fig. 1(a). In each one of the cavities we single out a quantum field mode with complex amplitude operator $a$ (left cavity) and $b$ (right cavity), both having the same frequency $\omega$. Initially mode $b$ is in the vacuum state, while mode $a$ is in an arbitrary state. We assume that losses are negligible during the detection. The coupling between the cavities induced by the beam splitter can be described by the Hamiltonian (in units $\hbar=1$ )

$$
H_{\kappa}=\kappa\left(a^{\dagger} b+a b^{\dagger}\right)
$$

where $\kappa$ is a parameter depending on the reflectivity of the beam splitter. Due to this coupling the photons initially in the left cavity can pass from one cavity to the other. If no other couplings were present, after a time $t=\pi /(2 \kappa)$ mode $a$ would be in vacuum and all the photons would be in mode $b$.

The atom is located in the right cavity. The field mode $b$ is resonant with the transition $|g\rangle \leftrightarrow|r\rangle$ between $|g\rangle$ and an auxiliary excited level $|r\rangle$, as shown in Fig. 1(b). In the 


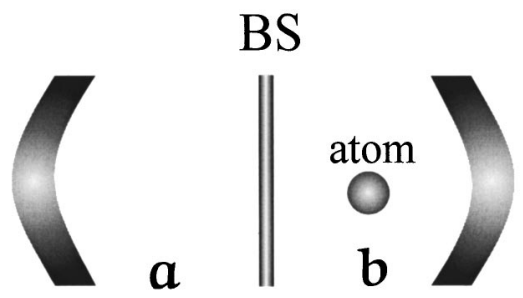

(a)

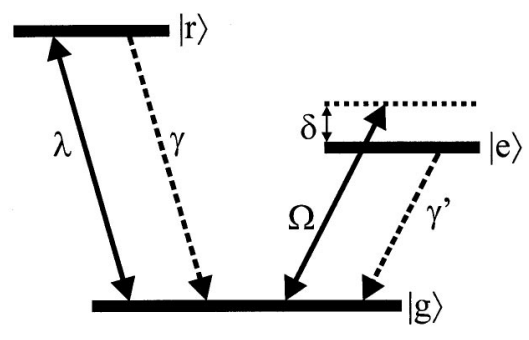

(b)

FIG. 1. (a) Outline of the arrangement for an interaction-free detection of the atomic state showing the two cavities coupled by a beam splitter BS and the two field modes $a$ (left cavity) and $b$ (right cavity). The atom is in the right cavity. (b) Diagram showing the relevant energy levels of the atom and their couplings.

electric-dipole and rotating-wave approximations this resonant interaction is described by the Hamiltonian

$$
H_{\lambda}=\lambda\left(|g\rangle\left\langle r\left|b^{\dagger}+\right| r\right\rangle\langle g| b\right),
$$

where $\lambda$ is the atom-field coupling constant. We assume that the level $|r\rangle$ is unstable and decays to $|g\rangle$ at a rate $\gamma$, for instance by the spontaneous emission of photons in a set of field modes. Other decaying mechanisms as well as other final states would also be valid.

Throughout this paper we shall work within the interaction picture, where the unperturbed Hamiltonian

$$
H_{0}=\omega\left(a^{\dagger} a+b^{\dagger} b\right)+\omega|r\rangle\left\langle r\left|+\omega_{f}\right| e\right\rangle\langle e|,
$$

with $\omega_{f}$ the frequency of the classical field, is removed by the unitary transformation $U=\exp \left(-i t H_{0}\right)$. Then, the evolution of the joint atom-field density matrix $\rho$ is

$$
\begin{aligned}
\dot{\rho}= & -i\left[H_{\kappa}+H_{\lambda}+H, \rho\right]-\frac{\gamma}{2}(|r\rangle\langle r|\rho+\rho| r\rangle\langle r| \\
& -2|g\rangle\langle r|\rho| r\rangle\langle g|),
\end{aligned}
$$

where $H$ represents the unobserved dynamics of the atom in the interaction picture and the last term stands for the spontaneous decay of the level $|r\rangle$ to $|g\rangle$.

This scenario includes well-known detection processes where the possible fluorescence caused by the $|g\rangle \leftrightarrow|r\rangle$ transition is monitored (quantum jumps, intermittent fluorescence, or electron shelving $[3,4])$. In such detection schemes there is an exchange of photons between the atom and the apparatus.
However, in this work we look for an atomic detection involving no processes of absorption and/or emission. This can occur if the transition $|g\rangle \leftrightarrow|r\rangle$ and the decay of the level $|r\rangle$ are fast enough. Then, if the atom is in $|g\rangle$ any photon in $b$ coming from mode $a$ through the beam splitter will be promptly and efficiently removed from modes $a$ and $b$. To this end, we assume that the probability of spontaneous emission in field modes other than $b$ is much larger than the stimulated reemission in the same mode $b$ of the incident photon. For a single incident photon this will be the case, provided that $\gamma \gg \lambda$.

In such a case, the atom in $|g\rangle$ becomes an ideal continuous detector of the field transition from the left to the right cavity, or, in other words, the right cavity becomes a nearly perfect absorber or scatterer, and this continually projects back the field onto the left cavity [2,8] (quantum Zeno effect). Thus, all the photons tend to remain in the left cavity and this prevents their absorption.

On the other hand, if the atomic state is $|e\rangle$, the photons can pass freely from $a$ to $b$, and when $t=\pi /(2 \kappa)$ all photons are in the right cavity. Consequently, the presence or absence of photons in the left cavity would indicate that the atomic state is $|g\rangle$ or $|e\rangle$, respectively. This will occur without any absorption and/or emission of photons.

This possibility must be included in the solution of Eq. (2.4). Whether the detection takes place with or without photon exchange will be determined by the particular values of the parameters $\kappa, \lambda$, and $\gamma$. Instead of attempting a direct solution, we shall try to simplify Eq. (2.4) in accordance with the objective pursued. The preceding discussion suggests that $\lambda$ and $\gamma$ are larger than the frequencies associated with $H_{\kappa}$ and $H$, so that the time spent in the excitation and decay of $|r\rangle$ is short compared to the field transit time between cavities and the transition time between the atomic levels $|e\rangle$ and $|g\rangle$. This allows us to simplify the evolution equation by adiabatically eliminating the faster variables decaying at rate $\gamma$, which are all the matrix elements involving the auxiliary level $|r\rangle$. We can consider that such terms are at each instant in their steady-state values and follow adiabatically the slower variation of the other terms [4]. Moreover, we will assume also that $\gamma \gg \lambda$, which ensures that there will be no $|g\rangle \leftrightarrow|r\rangle$ transitions before the possible spontaneous decay of $|r\rangle$ takes place.

Under these conditions, Eq. (2.4) leads to [11]

$$
\dot{\rho}=-i\left[H_{\kappa}+H, \rho\right]-\frac{2 \lambda^{2}}{\gamma}\left(B^{\dagger} B \rho+\rho B^{\dagger} B-2 B \rho B^{\dagger}\right),
$$

where

$$
B=b|g\rangle\langle g| \text {. }
$$

From now on, the density matrix $\rho$ involves only the field modes $a$ and $b$ and the two atomic levels $|g\rangle$ and $|e\rangle$, since any dependence on $|r\rangle$ has been adiabatically removed.

Although all these approximations imply that the population of $|r\rangle$ is negligible, the rate of absorptions and emissions can still be very large, so further requirements are needed in order to prevent them. This can be achieved if $\lambda^{2} / \gamma$ is larger 
than the frequencies associated with $H_{\kappa}$ and $H$. In such a case, $\rho$ will be always in the steady state determined by the second term in Eq. (2.5),

$$
B^{\dagger} B \rho+\rho B^{\dagger} B-2 B \rho B^{\dagger}=0 .
$$

It can be seen that this is equivalent to saying that $\rho$ satisfies

$$
\rho=\Pi \rho \Pi,
$$

where

$$
\Pi=|0\rangle_{b}{ }_{b}\langle 0|| g\rangle\langle g|+| e\rangle\langle e|,
$$

and $|0\rangle_{b}$ is the vacuum state in mode $b$.

The requirement (2.8) does not mean that the atom-field dynamics is halted: the system can still evolve under the action of $H_{\kappa}+H$, but in such a way that condition (2.8) is continuously satisfied. This can be dealt with by considering that actually the Hamiltonian is not $H_{\kappa}+H$, but a different one $\widetilde{H}_{\kappa}+\widetilde{H}$ embodying condition (2.8). Such an effective Hamiltonian can be deduced from Eq. (2.5) as follows. If $\rho(t)$ and $\rho(t+d t)$ satisfy Eq. (2.8), we have

$$
\begin{aligned}
\rho(t+d t)= & \Pi\left\{\rho(t)-i\left[H_{\kappa}+H, \Pi \rho(t) \Pi\right] d t\right\} \Pi=\rho(t) \\
& -i\left[\Pi\left(H_{\kappa}+H\right) \Pi, \rho(t)\right] d t
\end{aligned}
$$

so that the evolution of $\rho$ can be written as

$$
\dot{\rho}=-i\left[\widetilde{H}_{\kappa}+\widetilde{H}, \rho\right] \text {, }
$$

where

$$
\begin{gathered}
\widetilde{H}=\Pi H \Pi, \\
\widetilde{H}_{\kappa}=\Pi H_{\kappa} \Pi=\kappa|e\rangle\langle e|\left(a^{\dagger} b+a b^{\dagger}\right) .
\end{gathered}
$$

If the initial state fulfills condition (2.8), then the solution of the evolution equation (2.11) satisfies it at any instant.

This is valid for any atomic Hamiltonian $H$, provided that the conditions leading to Eq. (2.11) are satisfied. For definiteness, we shall focus on an atomic Hamiltonian $H$ having two contributions:

$$
H=H_{\Omega}+V
$$

First, we consider that the transition $|g\rangle \leftrightarrow|e\rangle$ is driven by a classical field of frequency $\omega_{f}$ such that

$$
H_{\Omega}=\Omega(|g\rangle\langle e|+| e\rangle\langle g|)-\delta|e\rangle\langle e|,
$$

where $2 \Omega$ is the Rabi frequency and $\delta=\omega_{f}-\omega_{0}$ is the detuning [12], $\hbar \omega_{0}$ being the free atomic-energy difference between $|e\rangle$ and $|g\rangle$. According to the previous analysis, this term must be included in the evolution of the observed atom in the form

$$
\widetilde{H}_{\Omega}=\Pi H_{\Omega} \Pi=\Omega|0\rangle_{b}{ }_{b}\langle 0|(|g\rangle\langle e|+| e\rangle\langle g|)-\delta| e\rangle\langle e| .
$$

We also consider that the excited state $|e\rangle$ can spontaneously decay to the level $|g\rangle$ due to the interaction with the environment. Such a coupling can be described by an interaction Hamiltonian $V$ of the form

$$
V=\sum_{k} \eta_{k}\left(c_{k}|e\rangle\left\langle g\left|+c_{k}^{\dagger}\right| g\right\rangle\langle e|\right)
$$

where $\eta_{k}$ are coupling constants and $c_{k}$ and $c_{k}^{\dagger}$ are the annihilation and creation operators of the environment modes, assumed to be initially in vacuum. This term should be included in the evolution of the observed atom in the form

$$
\widetilde{V}=\Pi V \Pi=\sum_{k} \eta_{k}|0\rangle_{b}{ }_{b}\langle 0|\left(c_{k}|e\rangle\left\langle g\left|+c_{k}^{\dagger}\right| g\right\rangle\langle e|\right) .
$$

The usual procedures of elimination of the degrees of freedom represented by $c_{k}$ lead to the following evolution equation:

$$
\dot{\rho}=-i\left[\widetilde{H}_{\kappa}+\widetilde{H}_{\Omega}, \rho\right]-\frac{\gamma^{\prime}}{2}\left(A^{\dagger} A \rho+\rho A^{\dagger} A-2 A \rho A^{\dagger}\right),
$$

where

$$
A=\Pi|g\rangle\langle e|\Pi=| 0\rangle_{b b}\langle 0|| g\rangle\langle e|,
$$

and $\gamma^{\prime}$ is a constant.

This is the final form for the atom-apparatus evolution during the detection. It can be noted that this atom-apparatus coupling is rather involved and does not resemble more standard procedures for continuous observation.

We can check that, as was intended, there is no photon absorption during the process. This is because the total photon number in modes $a$ and $b$ is a constant of the motion

$$
\frac{d}{d t}\left\langle\left(a^{\dagger} a+b^{\dagger} b\right)^{m}\right\rangle=0
$$

for any integer $m$. If a photon were absorbed in the $|g\rangle$ $\rightarrow|r\rangle$ transition, it would disappear definitively from modes $a$ and $b$ due to the high probability of the spontaneous decay of $|r\rangle(\gamma \gg \lambda)$.

Despite this, the field state can convey relevant information about the atomic state, since the evolution of modes $a$ and $b$ strongly depends on the level occupied by the atom. When the atom is in $|e\rangle$, the field can pass freely from mode $a$ to mode $b$. On the contrary, when the atom is in $|g\rangle$ the dynamics of $a$ and $b$ is completely inhibited. Thus, the field state provides a continuous measurement of the atomic level occupation with a time resolution of the order of $1 / \kappa$. Although this detection occurs without photon exchange; i.e., without forcing any internal transition on the atom, the evolution of the two-level atom is strongly modified, as we shall demonstrate in the next section.

Finally, we examine whether the relations between parameters required to prevent photon exchange are within the reach of current technology. These conditions are $\gamma \gg \lambda$ $\gg \kappa, \Omega, \gamma^{\prime}$ and $\lambda^{2} / \gamma \gg \kappa, \Omega, \gamma^{\prime}$. In order to dismiss photon losses from the double cavity, we can focus on microwave resonators and Rydberg atoms. In such a case, $\lambda$ can be of the order of $\lambda \sim 10^{6} \mathrm{~s}^{-1}$ [13], while the frequency $\kappa$ can be 
tuned at will [14]. For example, if $\kappa \sim 10^{3} \mathrm{~s}^{-1}$, the period of energy exchange between the cavities would be smaller than the photon lifetime within the cavities. Concerning $\gamma$, a suitable value would be $\gamma \sim 10^{8} \mathrm{~s}^{-1}$. All these values fulfill the mentioned conditions and are accessible to current experiments. Moreover, the feasibility of these parameters can be improved by involving additional auxiliary atomic levels, which increases the freedom to meet the requirements on the parameters without modifying the final conclusions. For instance, a suitable modification of the level scheme of Fig. 1(b) can be obtained if $|r\rangle$ is allowed to decay to another level different from $|g\rangle$, as is discussed in Ref. [11].

\section{DYNAMICS OF THE OBSERVED TWO-LEVEL ATOM}

The performance of the detection, as well as the alteration of the atomic evolution, depends on the relative weight of $\widetilde{H}_{\kappa}$ in comparison with the other terms in Eq. (2.18). If $\kappa$ is small, the atom evolves as if it were unobserved, while the field state carries no information about the atom. Due to the small time resolution, the apparatus will not be able to follow the faster internal evolution of the atom. On the contrary, if $\kappa$ is large we can expect an accurate monitoring, but the evolution of the atom will be significantly disturbed, being completely stopped in the limit of perfect detection (quantum Zeno effect). For intermediate situations, a meaningful observation is possible while the atom is allowed to evolve.

A full analytical solution seems difficult to obtain, mainly due to its highly nonlinear dependence on the complex amplitude operator $b$. Nevertheless, it is possible to obtain meaningful conclusions by examining the steady-state solution. For the sake of simplicity, we shall consider that the field modes contain only a single photon. In this case, the steady-state solution of Eq. (2.18) is (provided $\kappa \neq 0$ )

$$
\begin{aligned}
\rho_{g g}=\mathcal{N} & {\left[\left(\delta^{2}-\kappa^{2}\right)^{2}+\delta^{2}\left(\Omega^{2}+\gamma^{\prime 2} / 4\right)+\Omega^{2} \kappa^{2}\right]|1,0\rangle\langle 1,0|, } \\
\rho_{g e}= & \mathcal{N} \Omega \delta\left(\delta^{2}-\kappa^{2}+i \delta \gamma^{\prime} / 2\right)|1,0\rangle\langle 1,0|+\mathcal{N} \Omega \kappa\left(\delta^{2}-\kappa^{2}\right. \\
- & \left.\Omega^{2}+i \delta \gamma^{\prime} / 2\right)|1,0\rangle\langle 0,1|, \\
\rho_{e e}= & \mathcal{N} \Omega^{2} \delta^{2}|1,0\rangle\left\langle 1,0\left|+\mathcal{N} \Omega^{2}\left(\Omega^{2}+\kappa^{2}\right)\right| 0,1\right\rangle\langle 0,1| \\
& +\mathcal{N} \Omega^{2} \delta \kappa(|1,0\rangle\langle 0,1|+| 0,1\rangle\langle 1,0|)
\end{aligned}
$$

where

$$
\mathcal{N}=\frac{1}{\left(\Omega^{2}+\kappa^{2}\right)^{2}+\delta^{2}\left[\delta^{2}+2\left(\Omega^{2}-\kappa^{2}\right)+\gamma^{\prime 2} / 4\right]},
$$

and $\rho_{k \ell}=\langle k|\rho| \ell\rangle(k, \ell=e, g)$ are operators acting on the Hilbert space of the field modes, with $\left|n_{a}, n_{b}\right\rangle\left\langle n_{a}^{\prime}, n_{b}^{\prime}\right|$ the corresponding matrix elements in the photon-number basis.

First, we can examine to what extent the field state provides a measurement of the atomic-level occupation. According to the results of the preceding section, we can take the presence of the photon in the left cavity (where it was initially) as revealing that the atomic level is $|g\rangle$, while its presence in the right cavity can be interpreted as a signature of the occupation of the level $|e\rangle$. The explicit form of the total density matrix (3.1) allows us to calculate the probability of error $\mathcal{E}$ of this inference. Denoting by $P_{10 g}$ the prob- ability of finding the atom in $|g\rangle$ and the photon in $a$, and by $P_{01 e}$ the probability of the atom in $|e\rangle$ and the photon in $b$, the error probability is

$$
\mathcal{E}=1-P_{10 g}-P_{01 e}=\mathcal{N} \Omega^{2} \delta^{2} .
$$

The inference of the atomic level is always error free at resonance $\delta=0$. When $\delta$ is varied, the maximum for $\mathcal{E}$ occurs when $\delta= \pm \sqrt{\Omega^{2}+\kappa^{2}}$, taking the value

$$
\mathcal{E}_{\max }=\frac{\Omega^{2}}{4 \Omega^{2}+\gamma^{\prime 2} / 4},
$$

which, perhaps surprisingly, does not depend on $\kappa$. This shows that in the steady state the probability of a false inference is always less than $25 \%$, being negligible when $\Omega$ $\ll \gamma^{\prime}$, which is satisfied in a number of interesting situations.

Next, we turn our attention to the atomic dynamics. The atomic reduced density matrix $\rho_{\text {at }}$ has the matrix elements

$$
\begin{gathered}
\left\langle e\left|\rho_{\text {at }}\right| e\right\rangle=\mathcal{N} \Omega^{2}\left(\Omega^{2}+\kappa^{2}+\delta^{2}\right), \\
\left\langle e\left|\rho_{\text {at }}\right| g\right\rangle=\mathcal{N} \Omega \delta\left(\delta^{2}-\kappa^{2}-i \delta \gamma^{\prime} / 2\right), \\
\left\langle g\left|\rho_{\text {at }}\right| g\right\rangle=\mathcal{N}\left[\kappa^{2}\left(\kappa^{2}+\Omega^{2}\right)+\delta^{2}\left(\delta^{2}+\Omega^{2}-2 \kappa^{2}+\gamma^{\prime 2} / 4\right)\right] .
\end{gathered}
$$

It will be convenient to compare this expression with the steady state for the unobserved atom under the same conditions. Denoting the unobserved quantities by a superscript $(0)$, the evolution equation is

$$
\begin{aligned}
\dot{\rho}_{\mathrm{at}}^{(0)}= & -i\left[H_{\Omega}, \rho_{\mathrm{at}}^{(0)}\right]-\frac{\gamma^{\prime}}{2}\left(|e\rangle\left\langle e\left|\rho_{\mathrm{at}}^{(0)}+\rho_{\mathrm{at}}^{(0)}\right| e\right\rangle\langle e|\right. \\
& \left.-2|g\rangle\left\langle e\left|\rho_{\mathrm{at}}^{(0)}\right| e\right\rangle\langle g|\right),
\end{aligned}
$$

whose steady-state solution is

$$
\begin{aligned}
& \left\langle e\left|\rho_{\mathrm{at}}^{(0)}\right| e\right\rangle=\frac{\Omega^{2}}{\delta^{2}+2 \Omega^{2}+\gamma^{\prime 2} / 4}, \\
& \left\langle e\left|\rho_{\mathrm{at}}^{(0)}\right| g\right\rangle=\frac{\Omega\left(\delta-i \gamma^{\prime} / 2\right)}{\delta^{2}+2 \Omega^{2}+\gamma^{\prime 2} / 4}, \\
& \left\langle g\left|\rho_{\mathrm{at}}^{(0)}\right| g\right\rangle=\frac{\delta^{2}+\Omega^{2}+\gamma^{\prime 2} / 4}{\delta^{2}+2 \Omega^{2}+\gamma^{\prime 2} / 4} .
\end{aligned}
$$

It can be appreciated that there are significant differences between $\rho_{\text {at }}$ and $\rho_{\text {at }}^{(0)}$. In what follows we will briefly examine some of them.

\section{A. Excitation probability and width of the resonance}

First we compare the probability of occupation of the excited state $|e\rangle$. At resonance, this probability for the observed $P_{e}$ and unobserved $P_{e}^{(0)}$ atom is

$$
P_{e}=\frac{\Omega^{2}}{\Omega^{2}+\kappa^{2}}, \quad P_{e}^{(0)}=\frac{\Omega^{2}}{2 \Omega^{2}+\gamma^{\prime 2} / 4},
$$




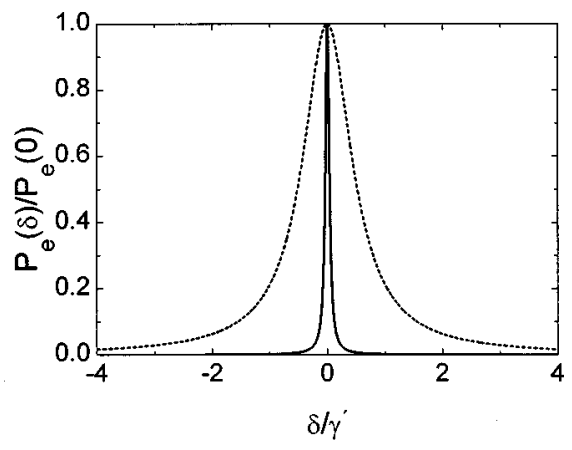

FIG. 2. Occupation probability of the excited state relative to its value at resonance $P_{e}(\delta) / P_{e}(0)$ as a function of $\delta / \gamma^{\prime}$ for $\kappa=\Omega$ $=0.1 \gamma^{\prime}$. The solid line is associated with the observed atom while the dashed line corresponds to the unobserved atom.

respectively. For the observed atom the population inversion can take any value. For instance, when $\Omega \gg \kappa$ the excitation probability can be arbitrarily close to 1 . Moreover, since $P_{e}$ does not depend on $\gamma^{\prime}$, we can have $P_{e} \simeq 1$, even if $\Omega \ll \gamma^{\prime}$. These two facts are in contrast to what occurs for the unobserved two-level atom. This behavior can be traced back to the evolution equation (2.18). The excitation of the atom tends to favor the presence of the photon in the right cavity, which in turn inhibits the spontaneous decay of $|e\rangle$.

If $\kappa \gg \Omega$, we have that $P_{e} \rightarrow 0$. This could be expected, since a very efficient monitoring tends to freeze the coherent transition $|g\rangle \leftrightarrow|e\rangle$, because of the randomization of the atomic dipole phase [11]. Then, the atom is led to its ground state by spontaneous decay.

The width of the resonance can be also quite different. It can be significantly narrower in the observed case than in the unobserved one. In Fig. 2 we have represented $P_{e}(\delta) / P_{e}(0)$ (solid line) together with $P_{e}^{(0)}(\delta) / P_{e}^{(0)}(0)$ (dashed line) as functions of $\delta / \gamma^{\prime}$ for $\Omega=\kappa=0.1 \gamma^{\prime}$, showing clearly this effect.

\section{B. Resonance splitting}

For some range of the parameters, the maximum inversion for the observed atom is not reached at resonance, unlike for the unobserved atom. This happens when $3 \kappa^{2}>\Omega^{2}$ $+\gamma^{\prime 2} / 4$, and in such a case, the atom has not one but two resonances located at

$$
\delta^{2}=\left(\kappa^{2}+\Omega^{2}\right)\left(\sqrt{\frac{4 \kappa^{2}-\gamma^{\prime 2} / 4}{\kappa^{2}+\Omega^{2}}}-1\right) .
$$

In Fig. 3 we have represented $P_{e}(\delta)$ (solid line) and $P_{e}^{(0)}(\delta)$ (dashed line) as functions of $\delta / \gamma^{\prime}$ for $\kappa=2 \gamma^{\prime}$ and $\Omega$ $=0.1 \gamma^{\prime}$. This splitting recalls the Autler-Townes doublet in a three-level atom when one of the transitions is driven by a strong enough field $[15,16]$. However, we stress that in our case the transition $|g\rangle \rightarrow|r\rangle$ has actually a negligible probability.

\section{Atomic coherence: Transparency at resonance}

Some further consequences of the observation can be found by examining the atomic coherence. The complex amplitude $\wp$ of the electric-dipole moment associated with the

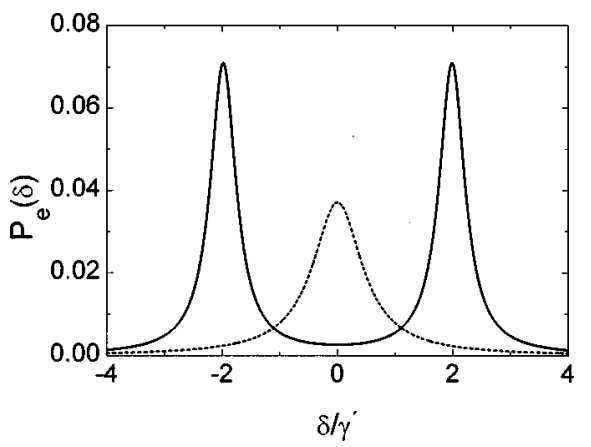

FIG. 3. Occupation probability of the excited state $P_{e}(\delta)$ as a function of $\delta / \gamma^{\prime}$ for $\kappa=2 \gamma^{\prime}$ and $\Omega=0.1 \gamma^{\prime}$. The solid line is associated with the observed atom while the dashed line corresponds to the unobserved atom.

transition $|g\rangle \leftrightarrow|e\rangle$ is proportional to the nondiagonal terms $\left\langle e\left|\rho_{\text {at }}\right| g\right\rangle$. For the observed atom we have

$$
\wp \propto \frac{\Omega \delta\left(\kappa^{2}-\delta^{2}+i \delta \gamma^{\prime} / 2\right)}{\left(\kappa^{2}+\Omega^{2}\right)^{2}+\delta^{2}\left[\delta^{2}+2\left(\Omega^{2}-\kappa^{2}\right)+\gamma^{\prime 2} / 4\right]},
$$

while in the unobserved case

$$
\wp^{(0)} \propto \frac{\Omega\left(-\delta+i \gamma^{\prime} / 2\right)}{\delta^{2}+2 \Omega^{2}+\gamma^{\prime 2} / 4} .
$$

If the classical field driving the transition is weak enough, we get the atomic linear polarizabilities

$$
\alpha \propto \frac{\delta\left(\kappa^{2}-\delta^{2}+i \delta \gamma^{\prime} / 2\right)}{\kappa^{4}+\delta^{2}\left(\delta^{2}-2 \kappa^{2}+\gamma^{\prime 2} / 4\right)}
$$

and

$$
\alpha^{(0)} \propto \frac{-\delta+i \gamma^{\prime} / 2}{\delta^{2}+\gamma^{\prime 2} / 4},
$$

respectively.

Perhaps the most noticeable effect reflected in these expressions is that $\wp$ and $\alpha$ vanish at resonance. This occurs always at $\delta=0$, irrespectively of the values taken by the rest of parameters. This implies that there is no absorption at resonance, precisely when it takes its maximum value for the unobserved atom. This absorption cancellation at $\delta=0$ produces two peaks in $\operatorname{Im}(\alpha)$ situated at $\delta= \pm \kappa$.

In Figs. 4 and 5 we have plotted the real and imaginary parts of $\alpha$ (solid line) and $\alpha^{(0)}$ (dashed line) as functions of $\delta / \gamma^{\prime}$ for $\kappa=0.2 \gamma^{\prime}$ (Fig. 4) and $\kappa=2 \gamma^{\prime}$ (Fig. 5). It can also be appreciated in these figures that $\operatorname{Re}(\alpha)$ has the usual relationship to $\operatorname{Im}(\alpha)$, with the particularity that the dispersion can be much steeper than usual in regions of transparency. As before, these phenomena recall equivalent behaviors in three-level atoms (electromagnetically-induced transparency and other related laser control of optical properties [16]).

Finally, we can note that the atomic coherence tends to vanish if $\kappa \rightarrow \infty$. Increasing the accuracy of the monitoring implies larger randomization of the atomic-dipole phase leading to $\wp, \alpha \rightarrow 0$. 
(a)

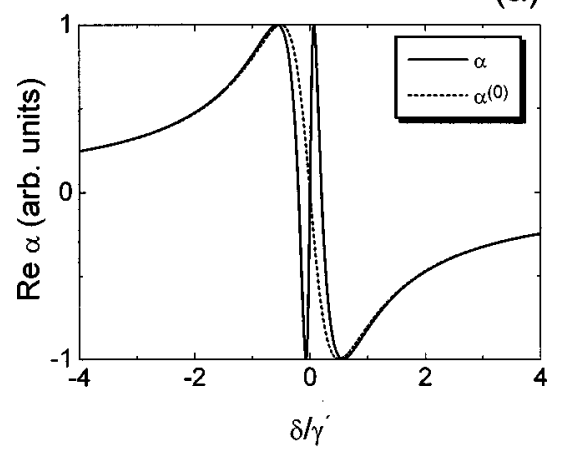

(b)

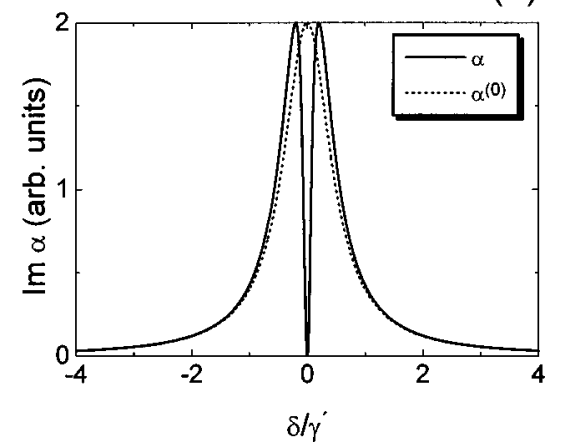

FIG. 4. Real (a) and imaginary (b) parts of the linear polarizability $\alpha$ in arbitrary units as a function of $\delta / \gamma^{\prime}$ for $\kappa=0.2 \gamma^{\prime}$. The solid line is associated with the observed atom while the dashed line corresponds to the unobserved atom.

\section{DRESSED-STATE PICTURE OF THE ATOM-APPARATUS SYSTEM}

The results of Sec. III suggest that the dynamics of the observed atom should admit a description in terms of a threelevel-like structure. However, such levels cannot be purely atomic. Although the original scheme in Fig. 1(b) involves three atomic levels, we have found that for some parameter regimes the population of $|r\rangle$ becomes negligible and the probability that the transition $|g\rangle \leftrightarrow|r\rangle$ actually takes place is as small as desired. Then, the atomic state can be very accurately described by the two-dimensional Hilbert space spanned by $|g\rangle$ and $|e\rangle$. This does not mean that level $|r\rangle$ has no influence on the evolution: such an influence is the unavoidable effective interaction present in any quantum detection process, including those based on seemingly interactionfree measurements.

Then, we have to resort to an energy-level scheme involving the atom and apparatus simultaneously. For the case considered in Sec. III, the atom-field system is spanned by the vectors $|0,1\rangle|e\rangle,|0,1\rangle|g\rangle,|1,0\rangle|e\rangle$, and $|1,0\rangle|g\rangle$. However, the dynamical constraint (2.8) implies that the state $|0,1\rangle|g\rangle$ cannot be occupied. Then, the actually allowed states are $|0,1\rangle|e\rangle,|1,0\rangle|e\rangle$, and $|1,0\rangle|g\rangle$. These states and their couplings, imposed by the evolution equation (2.18), are schematized in Fig. 6. This diagram should be helpful in understanding some of the effects shown in Sec. III.

In particular, the coupling between $|0,1\rangle|e\rangle$ and $|1,0\rangle|e\rangle$ causes the splitting of the upper level for the transition (a)

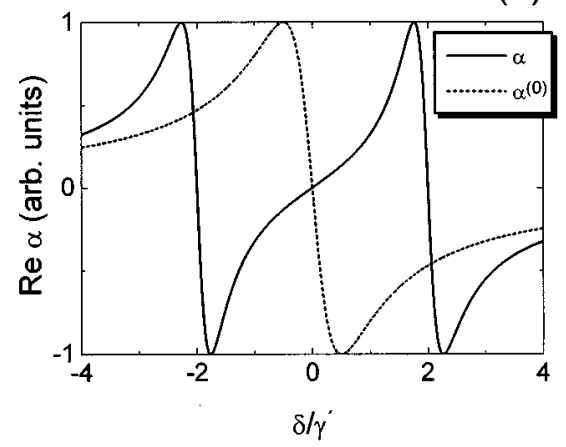

(b)

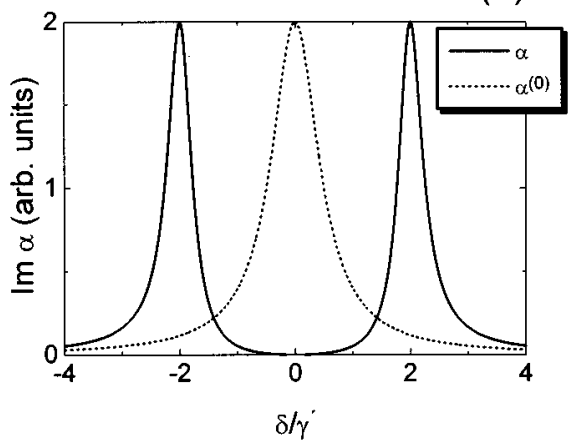

FIG. 5. Same as Fig. 4 for $\kappa=2 \gamma^{\prime}$.

driven by the classical field. This is formally the same mechanism leading to the Autler-Townes doublet in real three-level atoms, so this picture accounts for the splitting of the resonance found in the preceding section.

In order to account for the transparency at resonance we first note that when $\delta=0$ the state

$$
|\mathcal{D}\rangle=\frac{\kappa}{K}|1,0\rangle|g\rangle-\frac{\Omega}{K}|0,1\rangle|e\rangle,
$$

where $K=\sqrt{\Omega^{2}+\kappa^{2}}$, is electromagnetically decoupled from the rest of the states:

$$
\left(\widetilde{H}_{\kappa}+\widetilde{H}_{\Omega}\right)|\mathcal{D}\rangle=0 .
$$

At resonance, $\widetilde{H}_{\kappa}+\widetilde{H}_{\Omega}$ couples the state

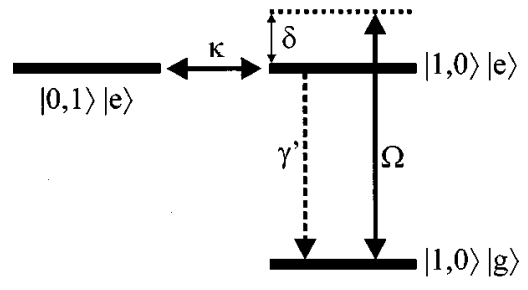

FIG. 6. Diagram showing the atom-field levels and their couplings when the field modes $a$ and $b$ contain a single photon. 


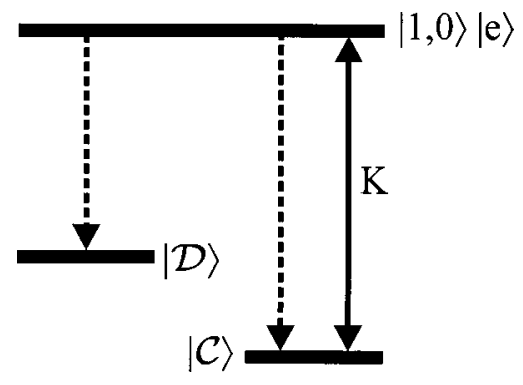

FIG. 7. Diagram showing the atom-field dressed levels at resonance when the field modes $a$ and $b$ contain a single photon.

$$
|\mathcal{C}\rangle=\frac{\Omega}{K}|1,0\rangle|g\rangle+\frac{\kappa}{K}|0,1\rangle|e\rangle,
$$

with the state $|1,0\rangle|e\rangle$ :

$$
\begin{gathered}
\left(\widetilde{H}_{\kappa}+\widetilde{H}_{\Omega}\right)|\mathcal{C}\rangle=K|1,0\rangle|e\rangle, \\
\left(\widetilde{H}_{\kappa}+\widetilde{H}_{\Omega}\right)|1,0\rangle|e\rangle=K|\mathcal{C}\rangle .
\end{gathered}
$$

In this basis, $|\mathcal{D}\rangle,|\mathcal{C}\rangle$, and $|1,0\rangle|e\rangle$, the action of the operator $A$ in Eq. (2.19), representing the spontaneous decay, is

$$
\begin{gathered}
A|\mathcal{D}\rangle=A|\mathcal{C}\rangle=0 \\
A|1,0\rangle|e\rangle=\frac{\kappa}{K}|\mathcal{D}\rangle+\frac{\Omega}{K}|\mathcal{C}\rangle .
\end{gathered}
$$

Then, when $\delta=0$ the diagram in Fig. 6 can be replaced by the level structure in Fig. 7.

During the joint atom-field evolution, the spontaneous decay of $|1,0\rangle|e\rangle$ populates $|\mathcal{D}\rangle$, while there is no process de- populating it. Then the occupation of $|\mathcal{D}\rangle$ grows, and when the steady state is reached the atom-field system is entirely in the pure state $|\mathcal{D}\rangle$, as can be checked in Eq. (3.1) for $\delta=0$. This is a dark or trapped state, in which the atomic-dipole moment vanishes. A similar situation is well known in threelevel atoms [16]. However, although the process is formally the same, the meaning of the levels involved is completely different.

Finally, we can show that this dressed-state picture explains the arbitrary excitation probability at resonance. The atomic excited level $|e\rangle$ is present in the upper level in Fig. 7 as well as in the dark state $|\mathcal{D}\rangle$. When the system reaches the steady state $|\mathcal{D}\rangle$, the level $|e\rangle$ is populated with probability $\Omega^{2} / K^{2}$.

\section{CONCLUSIONS}

We have analyzed an optical scheme to monitor the atomic state without photon exchange between the apparatus and the atom; i.e., without inducing any internal atomic transition. This is an example of continuous seemingly interaction-free detection. This quantum detection process implies constraints in the object-apparatus system moulding their joint evolution, which leads to an effective interaction that disturbs the original dynamics of the atom in a nontrivial way.

The atomic reduced dynamics shows relevant features (arbitrary population inversion in the steady state, for instance), which cannot be framed within the evolution of a two-level atom. Some other phenomena found are similar to known effects in three-level atoms (resonance splitting, transparency at resonance), despite the fact that only two atomic levels are involved. This behavior has been explained resorting to the dressed-atom-apparatus energy levels.
[1] W. M. Itano, D. J. Heinzen, J. J. Bollinger, and D. Wineland, Phys. Rev. A 41, 2295 (1990); S. M. Chumakov, K.-E. Hellwig, and A. L. Rivera, Phys. Lett. A 197, 73 (1995); R. Onofrio and L. Viola, Phys. Rev. A 56, 39 (1997).

[2] D. Home and M. A. B. Whitaker, Ann. Phys. (N.Y.) 258, 237 (1997).

[3] W. Nagourney, J. Sandberg, and H. Dehmelt, Phys. Rev. Lett. 56, 2797 (1986); T. Sauter, W. Neuhauser, R. Blatt, and P. E. Toschek, ibid. 57, 1696 (1986); J. C. Bergquist, R. G. Hulet, W. M. Itano, and D. J. Wineland, ibid. 57, 1699 (1986); V. Frerichs and A. Schenzle, Phys. Rev. A 44, 1962 (1991); E. Block and P. R. Berman, ibid. 44, 1466 (1991).

[4] H. J. Kimble, R. J. Cook, and A. L. Wells, Phys. Rev. A 34, 3190 (1986); M. J. Gagen and G. J. Milburn, ibid. 47, 1467 (1993).

[5] Y. Aharonov and M. Vardi, Phys. Rev. D 21, 2235 (1980); T. P. Altenmüller and A. Schenzle, Phys. Rev. A 48, 70 (1993).

[6] A. Luis and L. L. Sánchez-Soto, Phys. Lett. A 244, 211 (1998).

[7] A. C. Elitzur and L. Vaidman, Found. Phys. 23, 987 (1993); L. Vaidman, Quantum Opt. 6, 119 (1994); R. H. Dicke, Am. J. Phys. 49, 925 (1981).

[8] P. Kwiat, H. Weinfurter, T. Herzog, and A. Zeilinger, Phys.
Rev. Lett. 74, 4763 (1995); P. Kwiat, H. Weinfurter, T. Herzog, A. Zeilinger, and M. Kasevich, Ann. (N.Y.) Acad. Sci. 755, 383 (1995).

[9] M. Pavičić, Phys. Lett. A 223, 241 (1996).

[10] A. Luis and L. L. Sánchez-Soto, Phys. Rev. A 58, 836 (1998).

[11] A. Luis and L. L. Sánchez-Soto, Phys. Lett. A 252, 130 (1999).

[12] H. I. Yoo and J. H. Eberly, Phys. Rep. 118, 329 (1985); C. Cohen-Tannoudji, B. Diu, and F. Lalöe, Quantum Mechanics (John Wiley and Sons, New York, 1977), complement $F_{I V}$.

[13] B. W. Shore and P. L. Knight, J. Mod. Opt. 40, 1195 (1993); G. Rempe, H. Walther, and N. Klein, Phys. Rev. Lett. 58, 353 (1987); G. Rempe, F. Schmidt-Kaler, and H. Walther, ibid. 64, 2783 (1990); M. Brune, P. Nussenzveig, F. Schmidt-Kaler, F. Bernardot, A. Maali, J. M. Raimond, and S. Haroche, ibid. 72, 3339 (1994); L. Davidovich, M. Brune, J. M. Raimond, and S. Haroche, Phys. Rev. A 53, 1295 (1996).

[14] J. M. Raimond, M. Brune, and S. Haroche, Phys. Rev. Lett. 79, 1964 (1997).

[15] S. H. Autler and C. H. Townes, Phys. Rev. 100, 703 (1955); D. E. Nitz, A. V. Smith, M. D. Levenson, and S. J. Smith, Phys. Rev. A 24, 288 (1981).

[16] J. P. Marangos, J. Mod. Opt. 45, 471 (1998), and references therein. 